(c) 2007 International Press

Adv. Theor. Math. Phys. 11 (2007) 65-89

\title{
Geometric transitions and mixed Hodge structures
}

\author{
Duiliu-Emanuel Diaconescu ${ }^{1}$, Ron Donagi ${ }^{2}$, and Tony Pantev ${ }^{2}$
}

${ }^{1}$ New High Energy Theory Center, Rutgers University, 126 Frelinghuysen Road, Piscataway, NJ 08854, USA

${ }^{2}$ Department of Mathematics, University of Pennsylvania, David Rittenhouse Lab., 209 South 33rd Street, Philadelphia, PA 19104-6395, USA

\begin{abstract}
We formulate and prove a $\mathbf{B}$-model disc level large $N$ duality result for general conifold transitions between compact Calabi-Yau spaces using degenerations of Hodge structures.
\end{abstract}

\section{Introduction}

Large $N$ duality is a relation between open and closed string theories on two different Calabi-Yau manifolds connected by an extremal transition [1]. This relation was originally formulated in the context of topological A-model for a local conifold transition [1] and was extended to more general noncompact toric Calabi-Yau manifolds in $[2-5]$.

e-print archive: http://lanl.arXiv.org/abs/hep-th/0506195 
This paper is part of a long-term project aimed at understanding large $N$ duality for extremal transitions between compact Calabi-Yau manifolds. A first step in this direction has been made in [6] for topological A-models. Here, we will be concerned with large $N$ duality in the topological B-model. Open-closed duality for topological B-strings was first developed in $[7,8]$ for a special class of noncompact toric Calabi-Yau manifolds employing a remarkable relation between holomorphic Chern-Simons theory and random matrix models.

In contrast with the A-model, the topological B-model on compact Calabi-Yau spaces has not been given so far a rigorous mathematical description. However, it is well known that the genus zero topological closed string amplitudes can be expressed in Hodge theoretic terms using the formalism of special geometry. On the other hand, disc level topological open string amplitudes associated to D-branes wrapping curves in Calabi-Yau threefolds can also be given a geometric interpretation in terms of Abel-Jacobi maps [9-12]. Higher genus amplitudes do not have a pure geometric interpretation. In principle one would have to quantize Kodaira-Spencer theory coupled to holomorphic Chern-Simons theory on a compact CalabiYau space, which is a very hard task at best.

In this paper, we will formulate and prove a first order B-model duality statement for general conifold transitions between compact Calabi-Yau spaces. By first order duality, we mean a correspondence between topological disc amplitudes on the open string side and first-order terms in a suitable expansion of the holomorphic prepotential on the closed string side. The expansion is taken around an appropriate stratum parameterizing nodal Calabi-Yau spaces that admit a projective crepant resolution.

Using special geometry, in Section 2 we show that the first-order terms in this expansion admit a intrinsic geometric interpretation in terms of degenerations of Hodge structures. In Section 3, we will show that the first order duality statement follows from a Hodge theoretic result relating two different mixed Hodge structures. The main element in the proof is the ClemensSchmid exact sequence.

A connection between mixed Hodge structures and B-model topological disc amplitudes on toric Calabi-Yau manifolds has been previously developed in [13-15]. This machinery has been applied to first order large $N$ duality for toric Calabi-Yau manifolds in [16]. Our approach is different and can be used to extend the B-model large $N$ duality beyond disc level. Some progress along these lines for an interesting class of noncompact transitions is reported in the companion paper [17]. 


\section{B-Model transitions and periods}

In this section, we discuss the first order behavior of $\mathbf{B}$-model geometric transitions associated to conifold singularities of Calabi-Yau spaces. For us a Calabi-Yau space will be a (possibly singular) complex Gorenstein quasiprojective variety $X$ which has a trivial canonical class. In addition, in the singular case we will require that $X$ has a Kähler crepant resolution.

This convention is somewhat broader than the usual notion of a CalabiYau space used in physics, where one requires that $X$ is a complex analytic space equipped with a Ricci flat Kähler metric. We will be primarily interested in moduli spaces parameterizing Calabi-Yau structures. These moduli spaces can have different components corresponding to different sets of values of the topological invariants of $X$. A geometric (or extremal) transition is a process connecting two connected components of the moduli space through a degeneration. Schematically an extremal transition is captured in a diagram

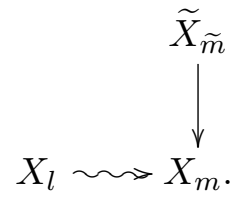

where $X_{l}$ is a smooth Calabi-Yau, $X_{l} \leadsto X_{m}$ is a degeneration of $X_{l}$ to a Calabi-Yau variety $X_{m}$ having only ordinary double points, and $\widetilde{X}_{\widetilde{m}} \rightarrow X_{m}$ is a crepant quasi-projective resolution of $X_{m}$.

The standard example of such a situation is the local conifold transition:

Example 2.1. Take $\left\{X_{\mu}\right\}_{\mu \in \mathbb{C}}$ to be the one parameter family of 3-dimensional affine quadrics $X_{\mu}=\left\{(x, y, z, w) \in \mathbb{C}^{4} \mid x y-z w=\mu\right\}$. When $\mu \rightarrow 0$ we get a degeneration $X_{\mu} \sim X_{0}$ of $X_{\mu}$ to the 3 -dimensional quadratic cone $x y=z w$. To complete this degeneration to a transition

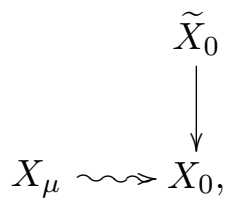

we take $\widetilde{X}_{0}$ to be one of the small resolutions of $X_{0}$, i.e., the blow-up of $X_{0}$ along the Weil divisor in $X_{0}$ corresponding to one of the two rulings in the base $\mathbb{P}^{1} \times \mathbb{P}^{1}$ of the cone. For future reference, we note also that as an abstract variety $\widetilde{X}_{0}$ is isomorphic to the total space of $\mathcal{O}(-1) \oplus \mathcal{O}(-1) \rightarrow \mathbb{P}^{1}$, and the map $\widetilde{X}_{0} \rightarrow X_{0}$ is the natural map contracting the zero section of $\mathcal{O}(-1) \oplus \mathcal{O}(-1)$. 
The geometric transition from $X_{l}$ to $\widetilde{X}_{\tilde{m}}$ changes the topology. Indeed, the process of degenerating $X_{l}$ to $X_{m}$ collapses some 3 -spheres in $X_{l}$ to the singular points of $X_{m}$. These spheres are the vanishing cycles of the degeneration $X_{l} \leadsto X_{m}$. On the other hand, the small resolution $\widetilde{X}_{\widetilde{m}} \rightarrow X_{m}$ replaces each singular point of $X_{m}$ by a copy of $\mathbb{P}^{1} \cong S^{2}$. So in the passage from $X_{l}$ to $\widetilde{X}_{m}$ we deleted some 3 -spheres from $X_{l}$ and glued 2-spheres in their place. It is important to note that this process not only changes the topology but also alters the type of geometry of the Calabi-Yau spaces in question. More precisely the transition interchanges holomorphic and symplectic data: the exceptional $\mathbb{P}^{1}$ 's in $\widetilde{X}_{\widetilde{m}}$ are holomorphic curves, and conjecturally the vanishing 3-spheres in $X_{l}$ can be chosen to be special Lagrangian submanifolds for the Kähler form on $X_{l}$. In particular, in an A-model transition one expects a relationship between open Gromov-Witten invariants on $X_{l}$ (with boundaries on the vanishing 3-spheres) and closed Gromov-Witten invariants on $\widetilde{X}_{m}$. The precise form of such a relationship is the content of the A-model large $N$ duality which has been extensively analyzed in the literature, see $[3,4,6]$ and references therein. Here, we propose a geometric description for the $\mathbf{B}$-model counterpart of the large $N$ duality and investigate some of the mathematical and physical consequences of our proposal.

Suppose $\boldsymbol{L}$ is a fixed component of the moduli space of Calabi-Yau threefolds with at most ODP singularities. Given a point $l \in \boldsymbol{L}$ we write $X_{l}$ for the corresponding Calabi-Yau. We will always assume that for a general $l \in \boldsymbol{L}$, the variety $X_{l}$ is a smooth (compact or noncompact) Calabi-Yau threefold. In examples, we will often take $X_{l}$ to be complete intersections in some toric variety since we want to keep track of the family of $\mathbf{A}$-models mirror to the topological B-models specified by the $X_{l}$ 's.

More precisely, we will look at the subvariety of $\boldsymbol{L}$ parameterizing singular threefolds with ordinary double points which admit a crepant projective resolution. Let $\boldsymbol{M}$ be a component of this subvariety and let $v$ denote the number of ODPs of $X_{m}$, for a general $m \in \boldsymbol{M}$. In particular on a nearby smooth $X_{l}$, we have a collection of $v$ embedded Lagrangian 3-spheres $L_{1}, \ldots, L_{v}$ whose homology classes $\left[L_{1}\right], \ldots,\left[L_{v}\right] \in H_{3}\left(X_{m}, \mathbb{Z}\right)$ vanish under a deformation $X_{l} \leadsto X_{m}$. Recall that [18, Theorem 2.9] in order for $X_{m}$ to admit a projective small resolution, we must have at least one good relation among $\left[L_{1}\right], \ldots,\left[L_{v}\right]$. That is, in $H_{3}\left(X_{l}, \mathbb{Z}\right)$ we must have a relation of the form

$$
\sum_{i=1}^{v} c_{i}\left[L_{i}\right]=0 \in H_{3}\left(X_{l}, \mathbb{Z}\right), \quad \text { with } c_{i} \neq 0 \text { for all } i=1, \ldots, v
$$


Assuming that this is the case, let $r \geq 1$ denote the number of relations on the vanishing cycles. Then for a fixed point $m \in M, X_{m}$ may have finitely many different projective small resolutions related by flops. This means that the moduli space $\widetilde{\boldsymbol{M}}$ of the resolution is a finite to one cover of the component $\boldsymbol{M}$. In the following, we will denote by $\rho: \widetilde{\boldsymbol{M}} \rightarrow \boldsymbol{M}$ the covering map and by $\widetilde{m}$ a point of $\widetilde{\boldsymbol{M}}$ which projects to $m \in \boldsymbol{M}$. Since above we have restricted our considerations to the moduli space of Calabi-Yau threefolds with at most isolated ODP singularities, this cover is unramified. The branching points of the cover would correspond to singular threefolds with more complicated singularities which have been excluded by our definition of the moduli space $\boldsymbol{L}$.

The exceptional locus of the resolution $\widetilde{X}_{\widetilde{m}} \rightarrow X_{m}$ consists of $v$ smooth $(-1,-1)$ rational curves $C_{1}, \ldots, C_{v}$ satisfying $v-r$ relations in $H_{2}\left(\widetilde{X}_{\widetilde{m}}, \mathbb{Z}\right)$. Moreover, we have (see e.g. [19]) the following relations among Betti numbers of $X_{m}$ and $\widetilde{X}_{\tilde{m}}$ :

$$
\begin{aligned}
& b_{2}\left(\widetilde{X}_{\widetilde{m}}\right)=b_{2}\left(X_{m}\right)+r=b_{2}\left(X_{l}\right)+r, \\
& b_{3}\left(\widetilde{X}_{\tilde{m}}\right)=b_{3}\left(X_{m}\right)-(v-r)=b_{3}\left(X_{l}\right)-2(v-r), \\
& b_{4}\left(\widetilde{X}_{\tilde{m}}\right)=b_{4}\left(X_{m}\right)=b_{4}\left(X_{l}\right)+r .
\end{aligned}
$$

Large $N$ duality conjectures a correspondence between topological string theories defined on the Calabi-Yau manifolds $X_{l}$ and $\widetilde{X}_{\widetilde{m}}$ related by a geometric transition. In this paper, we will study B-model transitions, in which case the conjecture predicts an equivalence between closed topological strings on $X_{l}$ and open-closed topological strings on $\widetilde{X}_{\widetilde{m}}$. In physical terms, the open-closed topological string theory on the small resolution $\widetilde{X}_{\widetilde{m}}$ is defined by wrapping $N_{i}$ B-branes on the exceptional curves $C_{i}$. It is by now well established that in a rigorous framework $\mathbf{B}$-branes should be described by derived objects. However, for the purposes of the present paper, it suffices to think of a B-brane as an algebraic cycle on $\widetilde{X}_{\widetilde{m}}$ of the form $\sum_{i=1}^{v} N_{i} C_{i}$. Furthermore, we will restrict our considerations to homologically trivial D-brane configurations, i.e.,

$$
\sum_{i=1}^{v} N_{i}\left[C_{i}\right]=0
$$

where $\left[C_{i}\right] \in H_{2}\left(\widetilde{X}_{\widetilde{m}}, \mathbb{Z}\right)$ denotes the homology class of $C_{i}$. In principle, the open-closed topological $\mathbf{B}$, model should be well-defined from a physical point of view for any values of the multiplicities $N_{i}$. However, it will become clear later that the above homology condition is required by large $N$ duality. While there is no a priori explanation for this condition in topological string theory, in physical superstring theory, this is a direct consequence 
of the Gauss law for Ramond-Ramond flux. It is quite interesting that the topological version of large $N$ duality still requires us to impose the physical Gauss law.

\section{$2.1 \quad$ Closed strings}

The central object of study of any topological string theory is the partition function, which is a generating functional for topological string amplitudes. The partition function of the closed topological B-model on $X_{l}$ can be written as

$$
\mathcal{F}_{X_{l}}^{c l}=\sum_{g=0}^{\infty} g_{s}^{2 g-2} \mathcal{F}_{X_{l}}^{g} .
$$

The genus $g$ free energy $\mathcal{F}_{X_{l}}^{g}$ is heuristically defined in terms of functional integrals over moduli spaces of maps from compact genus $g$ Riemann surfaces to $X_{l}$. In the B-model, the functional integral receives contributions only from degenerate maps, which sit on the boundary of the moduli space. For genus zero, the degenerate maps in question are constant maps, and the functional integral reduces to an ordinary integral on $X_{l}$ [20]. Moreover, the genus zero free energy depends holomorphically on the complex structure parameters of $X_{l}$. For higher genus, the degenerate maps have a more complicated structure and there is no rigorous mathematical formulation of topological amplitudes. In the following, we will restrict ourselves to genus zero topological strings.

Next we will explain the construction of the genus zero free energy $\mathcal{F}_{X_{l}}^{0}$ and its relation to the special geometry of the moduli space $\boldsymbol{L}$. Since in the B-model all physical correlators depend on the choice of a global holomorphic three-form, we have to introduce the enlarged moduli space $\boldsymbol{L}^{\prime}$ parameterizing pairs $\left(X_{l}, \Omega_{l}\right)$ where $\Omega_{l}$ is a nonzero global holomorphic three-form on $X_{l}$. Note that there is a complex holomorphic line bundle $\mathcal{L} \rightarrow \boldsymbol{L}$ so that the fiber $\mathcal{L}_{l}$ is the space of global holomorphic three-forms on $X_{l}$ for any point $l \in \boldsymbol{L}$. The enlarged moduli space $\boldsymbol{L}^{\prime}$ is isomorphic to the complement of the zero section in the total space of $\mathcal{L}$, hence it has the structure of a holomorphic principal $\mathbb{C}^{\times}$-bundle $\pi: \boldsymbol{L}^{\prime} \rightarrow \boldsymbol{L}$. Let us denote by $\boldsymbol{L}_{0}$ the open subspace of $\boldsymbol{L}$ parameterizing smooth varieties $X_{l}$, and by $\boldsymbol{L}_{0}^{\prime}$ its inverse image in $\boldsymbol{L}^{\prime}$. We also write $\boldsymbol{M}^{\prime}$ for the inverse image of $\boldsymbol{M}$ in $\boldsymbol{L}^{\prime}$, and $\widetilde{\boldsymbol{M}}^{\prime}$ for the enlarged moduli space of the resolution. Note that there is a finite to one unramified cover $\rho^{\prime}: \widetilde{\boldsymbol{M}}^{\prime} \rightarrow \boldsymbol{M}^{\prime}$.

Caution. The previous discussion is somewhat loose. For instance, the moduli $\boldsymbol{L}^{\prime}$ of pairs $\left(X_{l}, \Omega_{l}\right)$ is the total space of a line bundle $\mathcal{L} \rightarrow \boldsymbol{L}$ only if we 
view $\boldsymbol{L}$ as a stack. More importantly we need to make sure that $\mathcal{L} \rightarrow \boldsymbol{L}$ is a line bundle on $\boldsymbol{L}-\boldsymbol{L}_{0}$ as well. If we have a universal family $f: \mathcal{X} \rightarrow \boldsymbol{L}$, then $\mathcal{L}$ is the pushforward $f_{*} \omega_{\mathcal{X} / \boldsymbol{L}}$ of the relative dualizing sheaf, which is locally free by cohomology and base change. Indeed for this we only need to note that for a nodal Calabi-Yau $X_{m}$ we have $h^{0}\left(X_{m}, K_{X_{m}}\right)=h^{0}\left(X_{m}, \mathcal{O}\right)=1$ and $h^{1}\left(X_{m}, K_{X_{m}}\right)=h^{1}\left(X_{m}, \mathcal{O}\right)=0$.

Ideally one would like to define $\mathcal{F}_{X_{l}}^{0}$ as an intrinsic global geometric object on the moduli space $\boldsymbol{L}^{\prime}$ which can be locally described as a holomorphic function (for example, a section in a certain line bundle.) Unfortunately, there is no such intrinsic construction for $\mathcal{F}_{X_{l}}^{0}$. One can construct the three point function, or Yukawa coupling, as a global cubic form on $\boldsymbol{L}_{0}^{\prime}[21,22]$. The genus zero free energy can only be defined locally as a primitive of the Yukawa coupling. This description is of course ambiguous since the Yukawa coupling specifies only the third derivatives of the free energy. Therefore, in order to obtain a well-defined local function we have to make some choices. Using special geometry (see e.g. [21, 23]) one can show that a local primitive for the Yukawa coupling is determined by a choice of splitting of the third homology $H_{3}\left(X_{l}, \mathbb{Z}\right)$ into a direct sum $A \oplus B$ of maximal Lagrangian sublattices.

Recall that we have chosen $\boldsymbol{L}$ to be a component of a moduli space of Calabi-Yau threefolds with at most isolated ODP singularities. $\boldsymbol{M}$ is a subvariety of the discriminant parameterizing threefolds with a fixed number $v$ of ODPs which admit a crepant projective resolution. In generic situations, the $(v-r)$ codimensional subvariety $\boldsymbol{M}$ of the discriminant can be locally represented as the intersection locus of $v$ (local) branches of the discriminant. Therefore, we can choose an open subset $\mathcal{U} \subset \boldsymbol{L}$ so that $\mathcal{U} \cap \boldsymbol{M}$ is the intersection locus of a collection of Weil divisors $\boldsymbol{D}_{1}, \ldots, \boldsymbol{D}_{v}$ in $\mathcal{U}$ so that $v-r$ of them intersect transversely along $\boldsymbol{M}$. Moreover, $X_{l}$ with $l \in \mathcal{U}$ is singular if and only if $l$ is a point on

$$
\boldsymbol{D}=\boldsymbol{D}_{1}+\cdots+\boldsymbol{D}_{v}
$$

Let $\mathcal{U}^{\prime}$ denotes the inverse image of $\mathcal{U}$ in $\boldsymbol{L}^{\prime}$. In order to write down a local expression for the genus zero free energy, we have to introduce special coordinates $z^{\alpha}$ on $\mathcal{U}^{\prime}$ by choosing a symplectic basis of three-cycles $\left\{\gamma_{\alpha, l}, \gamma_{l}^{\alpha}\right\}$, $\alpha=1, \ldots h^{1,2}\left(X_{l}\right)+1$ on each threefold $X_{l}$, with $l \in \mathcal{U} \backslash \boldsymbol{D}$. The symplectic basis of cycles determines a splitting $H_{3}\left(X_{l}, \mathbb{Z}\right)=A_{l} \oplus B_{l}$ where $A_{l}, B_{l}$ are complementary maximal Lagrangian sublattices spanned by the cycles $\left\{\gamma_{\alpha, l}\right\}$ and $\left\{\gamma_{l}^{\alpha}\right\}$, respectively. Note that we have a monodromy transformation

$$
T_{i}: H_{3}\left(X_{l}, \mathbb{Z}\right) \longrightarrow H_{3}\left(X_{l}, \mathbb{Z}\right), \quad T_{i}(\Gamma)=\Gamma+\left\langle\Gamma, \xi_{i}\right\rangle \xi_{i}
$$


associated to each component $\boldsymbol{D}_{i}, i=1, \ldots, v$ of the discriminant in $\mathcal{U}$, where $\xi_{i} \in H_{3}\left(X_{l}, \mathbb{Z}\right)$ is the corresponding vanishing cycle. We have denoted by $\langle$,$\rangle the intersection pairing in H_{3}\left(X_{l}, \mathbb{Z}\right)$. In order to obtain single valued coordinates on $\mathcal{U}^{\prime}$, we have to choose the lattices of $A$-cycles so that $A_{l}$ is contained in the fixed locus of $T_{i}$ for each $i=1, \ldots, v$. Then the special projective coordinates are given by

$$
z^{\alpha}=\int_{\gamma_{\alpha, l}} \Omega_{X_{l}}
$$

where $\Omega_{X_{l}}$ is a global holomorphic three-form on $X_{l}$. Since the cycles $\gamma_{\alpha, l}$ are fixed by the monodromy transformations (2.2), the coordinates $z^{\alpha}$ extend as single valued holomorphic functions over the entire open set $\mathcal{U}^{\prime}$.

The genus zero free energy — or, adopting special geometry terminology, the holomorphic prepotential - is a multivalued holomorphic function on $\mathcal{U}^{\prime} \cap \boldsymbol{L}_{0}^{\prime}$ given by

$$
\mathcal{F}^{0}=\frac{1}{2} \sum_{\alpha=1}^{h^{1,2}\left(X_{l}\right)+1} z^{\alpha} \Pi_{\alpha}
$$

where

$$
\Pi_{\alpha}=\int_{\gamma_{l}^{\alpha}} \Omega_{X_{l}}
$$

are the periods of the holomorphic three-form on the $B$-cycles $\gamma_{l}^{\alpha}$. We also have the special geometry relations

$$
\Pi_{\alpha}=\frac{\partial \mathcal{F}^{0}}{\partial z^{\alpha}}
$$

which will be useful later in the paper.

\subsection{Open strings}

Let us now discuss the topological open-closed B-model on the small resolution $\widetilde{X}_{\widetilde{m}}$. In open-closed topological string theory, one would like to integrate over maps from genus $g$ Riemann surfaces with $h$ boundary components to $\widetilde{X}_{\widetilde{m}}$ mapping the boundary components to the exceptional curves $C_{i}$. The partition function of the theory is a generating functional of the form

$$
\mathcal{F}_{\widetilde{X}_{\tilde{m}},\left\{C_{i}\right\},\left\{N_{i}\right\}}=\sum_{g=0}^{\infty} \sum_{h_{1}, \ldots, h_{v}=0}^{\infty} g_{s}^{2 g-2+h} \mathcal{F}_{\widetilde{X}_{\tilde{m}},\left\{C_{i}\right\}}^{g,\left\{h_{i}\right\}} \prod_{i=1}^{v} N_{i}^{h_{i}}
$$

where $h=\sum_{i=1}^{v} h_{i}$. The coefficients $\mathcal{F}_{\widetilde{X}_{\widetilde{m}}, C_{i}}^{g,\left\{h_{i}\right\}}$ are heuristically defined in terms of functional integrals over maps from genus $g$ Riemann surfaces with $h$ boundary components to $\widetilde{X}_{m}$ mapping $h_{i}$ boundary components to the 
curve $C_{i}$. In the $\mathbf{B}$-model, they are expected to depend on complex moduli for the data $\left(\widetilde{X}_{\widetilde{m}}, C_{i}\right)$. Since the exceptional curves are rigid in the threefold, it follows that they only depend on the complex structure parameters of $\widetilde{X}_{\widetilde{m}}$.

According to [24], the open-string path integral localizes on degenerate maps, just as in the closed string situation. However, degenerate open string maps collapse a Riemann surface with boundary considered as a ribbon graph, to the corresponding graph embedded in $\widetilde{X}_{\tilde{m}}$. In particular, the degenerate maps can have a nontrivial structure even at genus zero. For this reason, there is no rigorous mathematical formulation of open-closed amplitudes except for $g=0$ and $h=0,1$ when degenerate maps are constant maps. In this paper, we will focus only on these two cases.

The term corresponding to $g=h=0$ in (2.5) is the genus zero closed string free energy $\mathcal{F}_{\widetilde{X}_{\tilde{m}}}^{0}$ which was introduced in Section 2.1. The term corresponding to $g=0, h=1$ represents the disc open string free energy which is determined by 3-dimensional chain integrals of the holomorphic three-form $\Omega_{\widetilde{X}_{\tilde{m}}}$. To explain this construction recall that we are considering D-brane configurations satisfying the homology constraint (2.1). For a fixed $\widetilde{m}$, the solutions to this equation are in one-to-one correspondence with points in the lattice

$$
\Lambda_{\widetilde{m}}=\operatorname{ker}\left(H_{2}(C) \longrightarrow H_{2}\left(\widetilde{X}_{\tilde{m}}\right)\right),
$$

where $C=\cup_{i=1}^{v} C_{i}$. Recall that in our set-up, the points in $\boldsymbol{M}$ parameterize threefolds with isolated ODP singularities, so that the cover $\widetilde{\boldsymbol{M}} \rightarrow \boldsymbol{M}$ is unramified. Then the lattices $\Lambda_{\widetilde{m}}$ span a locally constant sheaf when $\widetilde{m}$ varies in $\widetilde{\boldsymbol{M}}$. A flat section of this sheaf parameterizes a pair $\left(\widetilde{X}_{\widetilde{m}}, C_{N, \widetilde{m}}\right)$ where $C_{N, \widetilde{m}}$ is a homologically trivial algebraic cycle on $\widetilde{X}_{\widetilde{m}}$ of the form $C_{N, \widetilde{m}}=\sum_{i=1}^{v} N_{i} C_{i}$.

Regarding $C_{N, \widetilde{m}}$ as a topological brane on $\widetilde{X}_{\widetilde{m}}$, the disc partition function is a period of the holomorphic three-form $\Omega_{\widetilde{X}_{\tilde{m}}}$ over a 3 -chain, defined as follows. Consider the relative homology sequence for a pair $\left(\widetilde{X}_{\tilde{m}}, C\right)$, with $\widetilde{X}_{\widetilde{m}}$ smooth

$$
0 \longrightarrow H_{3}\left(\widetilde{X}_{\widetilde{m}}, \mathbb{Z}\right) \longrightarrow H_{3}\left(\widetilde{X}_{\widetilde{m}}, C, \mathbb{Z}\right) \stackrel{\delta}{\longrightarrow} \Lambda_{\widetilde{m}} \longrightarrow 0 .
$$

Given a homologically trivial algebraic cycle $C_{N, \widetilde{m}}$ on $\widetilde{X}_{\widetilde{m}}$, we can find a relative cycle $\widetilde{\Gamma}_{N, \widetilde{m}} \in H_{3}\left(\widetilde{X}_{\widetilde{m}}, C\right)$ so that

$$
\delta \widetilde{\Gamma}_{N, \widetilde{m}}=C_{N, \widetilde{m}}
$$


According to $[10,12]$, for fixed $N_{i}$ satisfying the condition (2.1) the disc partition function is the holomorphic function (= the Griffiths normal function associated to $C_{N, \widetilde{m}}$ ) on the enlarged moduli space $\widetilde{\boldsymbol{M}}^{\prime}$ given by

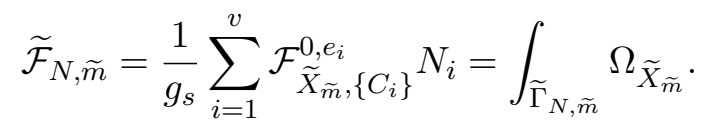

Here as usual we write $e_{i}$ for the $i$ th vector in the standard basis of the lattice $\mathbb{Z}^{\oplus v}$.

Note that the relative cycle $\widetilde{\Gamma}_{N}$ is only determined modulo elements in $H_{3}\left(\widetilde{X}_{\widetilde{m}}, \mathbb{Z}\right)$, therefore the superpotential (2.6) is defined modulo periods. In general, there is no preferred choice for a three-chain $\widetilde{\Gamma}_{N}$, hence we will regard this as a discrete ambiguity in the disc partition function.

\subsection{Duality}

Large $N$ duality predicts a correspondence between topological open and closed strings on a pair of Calabi-Yau threefolds related by an extremal transition. A precise mathematical statement has not been formulated for B-model transitions between compact Calabi-Yau threefolds. On general grounds, large $N$ duality is expected to relate genus zero open string amplitudes with $h$ boundary components on $\widetilde{M}^{\prime}$ to terms of order $h$ in the Taylor expansion of the closed string partition function on $\boldsymbol{L}^{\prime}$ near $\boldsymbol{M}^{\prime}$. In this subsection, our goal is to formulate a precise mathematical statement for genus zero terms with $h=0,1$.

We will show that for a certain choice of special coordinates $z^{\alpha}, \alpha=$ $1, \ldots, h^{1,2}\left(X_{l}\right)+1$ in a neighborhood of $\boldsymbol{M}^{\prime}$ in $\boldsymbol{L}^{\prime}$, the closed string prepotential $\mathcal{F}^{0}$ and its derivatives $\left(\frac{\partial \mathcal{F}^{0}}{\partial z^{\alpha}}\right)$ have well-defined limits $\mathcal{F}_{\boldsymbol{M}^{\prime}}^{0}$, $\left(\frac{\partial \mathcal{F}^{0}}{\partial z^{\alpha}}\right)_{\boldsymbol{M}^{\prime}}$ along $\boldsymbol{M}^{\prime}$. Moreover, the following relations hold

$$
\widetilde{\mathcal{F}^{0}}=\rho^{\prime *}\left(\mathcal{F}_{M^{\prime}}^{0}\right), \quad \widetilde{\mathcal{F}}_{i}=\rho^{\prime *}\left(\frac{\partial \mathcal{F}^{0}}{\partial z^{i}}\right)_{\boldsymbol{M}^{\prime}},
$$

where $\widetilde{\mathcal{F}} 0$ denotes the closed string prepotential on the moduli space $\widetilde{\boldsymbol{M}^{\prime}}$, $\widetilde{\mathcal{F}}^{i}, i=1, \ldots, v-r$ are open string superpotentials of the form (2.6), and $i=1, \ldots, v-r$ labels the normal directions to $\boldsymbol{M}^{\prime}$ in $\boldsymbol{L}^{\prime}$. The main point we would like to make is that these relations are a corollary of the following 
intrinsic geometric results which will be proved in the next section.

(i) For any $\widetilde{m} \in \widetilde{\boldsymbol{M}}$, and $m \in \boldsymbol{M}$ so that $m=\rho(\widetilde{m})$, the contraction map $\left(\widetilde{X}_{\tilde{m}}, C\right) \rightarrow\left(X_{m}, \operatorname{Sing}\left(X_{m}\right)\right)$ induces an isomorphism

$$
H_{3}\left(\widetilde{X}_{\widetilde{m}}, C, \mathbb{Z}\right) \simeq H_{3}\left(X_{m}, \mathbb{Z}\right) .
$$

Choose a retraction map ret $: \mathcal{X} \rightarrow \mathcal{X}_{\mid M}$ from the universal family on $\boldsymbol{L}$ onto the universal family on $\boldsymbol{M}$. (This can always be done in a neighborhood of a point $m \in \boldsymbol{M}$.) Suppose $\Gamma$ is a multivalued section of the sheaf of third homology groups $H_{3}\left(X_{l}, \mathbb{Z}\right)$ on $\boldsymbol{L}$, then the image of $\Gamma$ under ret induces a multivalued section $\widetilde{\Gamma}$ of the sheaf of relative homology groups $H_{3}\left(\widetilde{X}_{\widetilde{m}}, C ; \mathbb{Z}\right)$ on $\widetilde{\boldsymbol{M}}$.

(ii) Let

$$
\Pi=\int_{\Gamma_{l}} \Omega_{X_{l}}, \quad \widetilde{\Pi}=\int_{\widetilde{\Gamma}_{\widetilde{m}}} \Omega_{\widetilde{X}_{\widetilde{m}}}
$$

be the periods of the respective holomorphic 3-forms, viewed as multivalued holomorphic functions on $\boldsymbol{L}_{0}^{\prime}$ and $\widetilde{\boldsymbol{M}}^{\prime}$. Then $\Pi$ induces a multivalued holomorphic function $\Pi_{\boldsymbol{M}^{\prime}}$ on $\boldsymbol{M}^{\prime}$ and

$$
\widetilde{\Pi}=\rho^{\prime *}\left(\Pi_{M^{\prime}}\right) \text {. }
$$

(iii) For any points $l \in \boldsymbol{L}_{0}, \widetilde{m} \in \widetilde{\boldsymbol{M}}$, such that $\operatorname{ret}(l)=\rho(\widetilde{m})$, there is a commutative diagram of the form

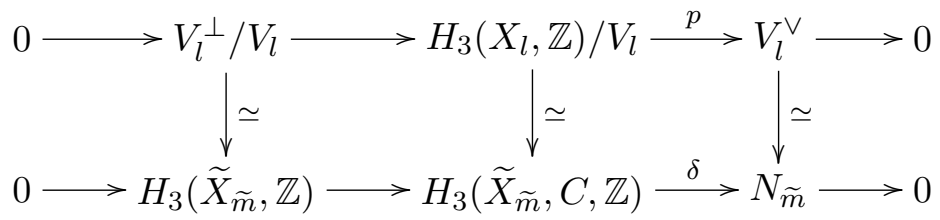

where the rows are exact and the vertical arrows are isomorphisms. $V_{l}$ is the lattice of vanishing cycles in $H_{3}\left(X_{l}\right)$ and $V_{l}^{\perp}$ denotes the orthogonal lattice with respect to the intersection pairing. The map $p: H_{3}\left(X_{l}, \mathbb{Z}\right) / V_{l} \rightarrow V_{l}^{\vee}$ is given by $p(\bar{\Gamma})=\langle\bar{\Gamma}, \bullet\rangle$ which is well-defined because $V_{l}$ is isotropic with respect to the intersection pairing.

The claim (i) follows from the definition of relative homology groups. The proof of assertions (ii) and (iii) will be given in section 3. In the remaining part of this section, we will explain how these statements lead to large $N$ duality up to first order. Recall from Section 2.1 that for $l \in \boldsymbol{L}_{0}$ the genus zero closed string partition function can be written locally in terms of splitting of the third homology groups $H_{3}\left(X_{l}, \mathbb{Z}\right)$ into a direct sum of two Lagrangian sublattices. Restricting to an open subset $\mathcal{U}^{\prime}$ of $\boldsymbol{L}^{\prime}$, suppose we 
can choose the Lagrangian sublattices $A_{l}, B_{l}$ for a smooth $X_{l}$ so that

$$
A_{l}=V_{l} \oplus \widetilde{A}_{l}, \quad B_{l}=U_{l} \oplus \widetilde{B}_{l}
$$

subject to the following conditions:

(a) $A_{l}$ is contained in the fixed locus of the monodromy transformations (2.2).

(b) $V_{l}$ is orthogonal to $\widetilde{B}_{l}$ and $U_{l}$ is orthogonal to $\widetilde{A}_{l}$ with respect to the intersection pairing.

More concretely, this means that we choose the symplectic basis of cycles so that $\left\{\gamma_{1, l}, \ldots, \gamma_{v-r, l}\right\}$ generate the lattice $V_{l}$ of vanishing cycles. Then $U_{l}$ is generated by the dual cycles $\left\{\gamma_{l}^{1}, \ldots, \gamma_{l}^{v-r}\right\}$ and $V_{l}^{\perp}$ is generated by $\left\{\gamma_{\beta, l}, \gamma_{l}^{\alpha}\right\}$ for $\alpha=v-r+1, \ldots, h^{1,2}\left(X_{l}\right)+1$ and $\beta=1, \ldots, h^{1,2}\left(X_{l}\right)+1$. Although there is no such canonical choice, note that we can obtain a basis of $V_{l}$ by choosing any collection $\boldsymbol{D}_{i_{1}}, \ldots, \boldsymbol{D}_{i_{v-r}}$ of local components of the discriminant which intersect transversely along $\boldsymbol{M}$. Then we can complete this basis to a symplectic basis of cycles of $H_{3}\left(X_{l}, \mathbb{Z}\right)$.

Given such a basis of cycles on each smooth $X_{l}$, the commutative diagram (2.9) shows that the cycles $\left\{\gamma_{\alpha, l}, \gamma_{l}^{\alpha}\right\}$ for $\alpha=v-r+1, \ldots, h^{1,2}\left(X_{l}\right)+$ 1 induce a symplectic basis of cycles on $\widetilde{X}_{\widetilde{m}}$ for $\widetilde{m}$ in the open set $\widetilde{\mathcal{U}}=$ $\rho^{-1}(\mathcal{U} \cap \boldsymbol{M})$. Moreover, to each basis element $\gamma_{l}^{i}, i=1, \ldots, v-r$ we can associate an exceptional curve $C_{i, \widetilde{m}}$ on $\widetilde{X}_{\widetilde{m}}$ so that the $C_{i, \widetilde{m}}$ generate $\Lambda_{\widetilde{m}}$ for any $\widetilde{m} \in \widetilde{\mathcal{U}}$. The images of the cycles $\left\{\gamma_{l}^{1}, \ldots, \gamma_{l}^{v-r}\right\}$ in $H_{3}\left(V_{l}\right) / V_{l}$ induce relative three-cycles $\widetilde{\Gamma}_{1, \tilde{m}}, \ldots, \widetilde{\Gamma}_{v-r, \widetilde{m}}$ in $H_{3}\left(\widetilde{X}_{\tilde{m}}, C\right)$ so that

$$
\delta\left(\widetilde{\Gamma}_{i, \widetilde{m}}\right)=C_{i, \widetilde{m}}
$$

for $i=1, \ldots, v-r$. Then we have a well-defined closed string prepotential $\widetilde{\mathcal{F}}: \widetilde{\mathcal{U}}^{\prime} \rightarrow \mathbb{C}$ and open string superpotentials $\widetilde{\mathcal{F}}_{i}: \widetilde{\mathcal{U}}^{\prime} \rightarrow \mathbb{C}$

$$
\widetilde{\mathcal{F}}_{i}=\int_{\widetilde{\Gamma}_{i, \tilde{m}}} \Omega_{\widetilde{X}_{\widetilde{m}}}
$$

for each $C_{i, \widetilde{m}} \in \Lambda_{\widetilde{m}}$. The special geometry relations (2.3)-(2.4) together with statement (ii) above imply that the closed string prepotential $\mathcal{F}$ and its derivatives $\frac{\partial \mathcal{F}}{\partial z^{\alpha}}$ have well-defined limits $\mathcal{F}_{\boldsymbol{M}^{\prime}},\left(\frac{\partial \mathcal{F}}{\partial z^{\alpha}}\right)_{\boldsymbol{M}^{\prime}}$ at the points of $\mathcal{U}^{\prime} \cap \boldsymbol{M}^{\prime}$. The relations (2.7) follow from equation (2.8). Note that these relations are valid for any choice of a symplectic basis in $H_{3}\left(X_{l}\right)$ satisfying conditions $(a)$ and $(b)$ above. 


\section{Geometric transitions and mixed hodge structures}

In this section, we would like to prove statements (ii) and (iii). We will consider the following abstract situation. Let $\pi: \mathcal{X} \rightarrow \Delta$ be a one parameter family of projective Calabi-Yau threefolds over the unit disc $\Delta$. The generic fiber $X_{\mu}, \mu \in \Delta \backslash\{0\}$ is assumed to be smooth and the central fiber $X_{0}$ is a nodal threefold with ordinary double points $p_{1}, \ldots, p_{v}$. We will assume that over the complement of the central fiber, the family $\pi: \mathcal{X} \rightarrow \Delta$ is equipped with a globally defined relative holomorphic 3 -form, which restricts to a holomorphic volume form on each fiber. Moreover, we will assume that there is a smooth crepant projective resolution $\widetilde{X} \rightarrow X_{0}$. Such a family can be obtained for example by taking $\Delta$ to be a holomorphic disc in the moduli space $\boldsymbol{L}$ intersecting $\boldsymbol{M}$ transversely at the origin.

The restriction of the family $\mathcal{X} \rightarrow \Delta$ to the punctured disc $\Delta^{\times}=\Delta \backslash\{0\}$ is a family of smooth Calabi-Yau threefolds which determines a geometric variation of Hodge structures. To this data, we can associate a period map $\phi: \Delta^{\times} \rightarrow D / \mathfrak{M}[25]$, where $D$ is the classifying space of Hodge structures and $\mathfrak{M}$ is the monodromy group of the family.

The proof of claim (ii) is Hodge theoretic and is based on the nilpotent orbit theorem and the Clemens-Schmid exact sequence associated to the degeneration $\mathcal{X} \rightarrow \Delta$. In the process of proving claim (ii), we will also show that the Clemens-Schmid exact sequence implies claim (iii).

First observe that the nilpotent orbit theorem implies the existence of a well-defined limit of the periods of the holomorphic three-form at the origin $0 \in \Delta$. Suppose $\Gamma$ is a multivalued section of the sheaf of third homology groups $H_{3}\left(X_{s}\right)$ over $\Delta^{\times}$. Let

$$
\Pi(s)=\int_{\Gamma_{s}} \Omega_{X_{s}}
$$

be the period of the holomorphic three-form on $\Gamma_{s}$, for $s \neq 0$. $\Pi$ is a multivalued holomorphic function on the punctured disc $\Delta^{\times}=\Delta \backslash\{0\}$. Let

$$
\psi: \Delta^{*} \longrightarrow D^{\vee}, \quad \psi(s)=\exp \left(-\frac{\log s}{2 \pi i} N\right) \phi(s),
$$

be the modified period map $\phi$ with values in the compact dual $D^{\vee}$ of $D[25$, 26]. Here, $N=\log (T)=T$ - id is the logarithm of the monodromy transformation about the origin. The identity $\log (T)=T-$ id is equivalent to $(T-\mathrm{id})^{2}=0$ which holds for any ODP degeneration of threefolds. Indeed, this follows from the observation that if we write $T$ - id as a block matrix with respect to the decompositions (2.10), then the only nonzero block of $T-$ id is the one sending $U_{l}$ to $V_{l}$. 
According to the nilpotent orbit theorem [26], the map $\psi$ can be extended to a single valued holomorphic map

$$
\bar{\psi}: \Delta \longrightarrow D^{\vee}
$$

In particular, the multivalued map $\Pi: \Delta^{\times} \rightarrow \mathbb{C}$, which is a matrix coefficient of $\phi$, can be written as

$$
\Pi(s)=\bar{\Pi}(s)+\frac{1}{2 \pi i}(\log s) \eta(s)
$$

for some $\bar{\Pi}(s), \eta(s)$ single valued holomorphic functions of $s$ with $\eta(0)=0$. Later in the proof of relation (2.8) we will see that the value $\bar{\Pi}(0)$ admits an intrinsic description of in terms of the mixed Hodge structure of the semistable model of the degeneration $\mathcal{X} \rightarrow \Delta$.

The point $\bar{\psi}(0) \in D^{\vee}$ corresponds to the limiting mixed Hodge structure on the third cohomology group of a smooth fiber $H^{3}\left(X_{s}\right), s \neq 0$. On the other hand, the relative cohomology group $H^{3}(\tilde{X}, C)$ also carries a canonical mixed Hodge structure [27, 28]. The relation (2.8) that we wish to prove asserts that a period in the limiting mixed Hodge structure on $H^{3}\left(X_{s}\right)$ is equal to the corresponding period in the mixed Hodge structure on $H^{3}(\widetilde{X}, C)$. In particular, $(2.8)$ will follow if we can show that the natural map $H^{3}(\widetilde{X}, C) \rightarrow H^{3}\left(X_{s}\right)$ is an inclusion of mixed Hodge structures.

We claim that this follows from the Clemens-Schmid exact sequence. In order to formulate a more precise statement, let us first describe the mixed Hodge structure on $H^{3}(\widetilde{X}, C)$ and construct the Clemens-Schmid exact sequence for our degeneration.

Recall that a mixed Hodge structure on a vector space $H=H_{\mathbb{Z}} \otimes \mathbb{C}$ is defined by

- a descending Hodge filtration $\left\{F^{k}\right\}$, and

- an ascending weight filtration $\left\{W_{m}\right\}$, defined over $\mathbb{Q}$, so that for every $m$ the successive quotient $W_{m} / W_{m-1}$ has a pure Hodge structure of weight $m$ given by the induced filtration

$$
F^{k}\left(\frac{W_{m}}{W_{m-1}}\right)=\left(\frac{W_{m} \cap F^{k}}{W_{m-1} \cap F^{k}}\right) .
$$

The limiting mixed Hodge structure on the cohomology of a smooth fiber is defined by the Hodge filtration given by the nilpotent orbit [26] and by the 
monodromy weight filtration

$$
0 \subset W_{0} \subset W_{1} \subset \cdots \subset W_{5} \subset W_{6}=H^{3}\left(X_{s}\right),
$$

which is determined by the monodromy action on cohomology. In our case, $N^{2}=0$, and the monodromy weight filtration is very simple

$$
W_{3}=\operatorname{ker}(N), \quad W_{2}=\operatorname{im}(N) .
$$

Additional information about the limiting mixed Hodge structure can be obtained from the Clemens-Schmid exact sequence as we will see below.

To describe the mixed Hodge structure on $H^{3}(\widetilde{X}, C)$, consider the long exact sequence of the pair $(\widetilde{X}, C)$

$$
\cdots \longrightarrow H^{2}(\tilde{X}) \longrightarrow H^{2}(C) \longrightarrow H^{3}(\tilde{X}, C) \longrightarrow H^{3}(\tilde{X}) \longrightarrow 0
$$

where all cohomology groups have complex coefficients. The ascending weight filtration on $H^{3}(\widetilde{X}, C)$ is defined by

$$
\begin{aligned}
& W_{3}=H^{3}(\tilde{X}, C) \\
& W_{2}=\operatorname{Im}\left(H^{2}(C) \longrightarrow H^{3}(\widetilde{X}, C)\right) \simeq H^{2}(C) / \operatorname{im}\left(H^{2}(\widetilde{X}) \longrightarrow H^{2}(C)\right) \\
& W_{1}=0 .
\end{aligned}
$$

The Hodge filtration is the standard Hodge filtration on the cohomology of the quasi-projective variety $X \backslash C$. The relation between the mixed Hodge structures on $H^{3}\left(X_{s}\right)$ and $H^{3}(\widetilde{X}, C)$ will be extracted from the ClemensSchmid exact sequence, which is constructed in terms of a semistable model of the degeneration $\mathcal{X} \rightarrow \Delta$.

A semistable model for the family $\mathcal{X} \rightarrow \Delta$ is a new family $\overline{\mathcal{X}} \rightarrow \Delta$ which fits in a commutative diagram of the form

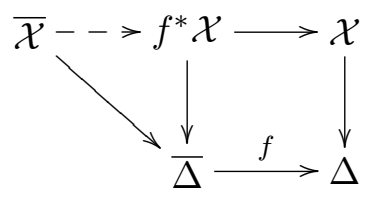

Here $\overline{\mathcal{X}}$ is assumed smooth, $f: \bar{\Delta} \rightarrow \Delta$ is a finite cover of $\Delta$ branched at the origin and the dashed arrow represents a birational map which is an isomorphism over the punctured disc $\bar{\Delta}^{\times}$. In addition, the central fiber $\bar{X} \equiv \bar{X}_{0}$ is required to be a normal crossing divisor in $\overline{\mathcal{X}}$ with smooth reduced irreducible components.

The cohomology of the central fiber $\bar{X}_{0}$ of the semistable degeneration can be equipped with a mixed Hodge structure, which can be described in 
terms of the Mayer-Vietoris spectral sequence. The Clemens-Schmid exact sequence is an exact sequence of mixed Hodge structures of the form

$$
\cdots \longrightarrow H_{1}\left(\bar{X}_{0}\right) \longrightarrow H^{3}\left(\bar{X}_{0}\right) \longrightarrow H^{3}\left(\bar{X}_{\bar{s}}\right) \stackrel{N}{\longrightarrow} H^{3}\left(\bar{X}_{\bar{s}}\right) \longrightarrow H_{3}\left(\bar{X}_{0}\right) \longrightarrow \cdots
$$

The Clemens-Schmid theorem [29] guarantees that all maps in this sequence are morphisms of mixed Hodge structures. The mixed Hodge structure on homology is induced by the mixed Hodge structure on cohomology using the universal coefficient formula.

Now, claim (ii) follows from the following two facts which will be proven in the rest of the section.

- $H^{3}\left(\bar{X}_{0}\right) \simeq \operatorname{ker}\left(N: H^{3}\left(\bar{X}_{\bar{s}}\right) \longrightarrow H^{3}\left(\bar{X}_{\bar{s}}\right)\right)$ as mixed Hodge structures. This follows from $H_{1}\left(\bar{X}_{0}\right)=0$.

- $H^{3}(\tilde{X}, C) \simeq H^{3}\left(\bar{X}_{0}\right)$ as mixed Hodge structures.

To begin with, let us construct the semistable degeneration for our family $\mathcal{X} \rightarrow \Delta$. Let $f: \bar{\Delta} \rightarrow \Delta$ be a double cover of the disc defined in local coordinates by $s=\bar{s}^{2}$, and let $f^{*} \mathcal{X}$ denote the pull-back of the family $\mathcal{X} \rightarrow \Delta$ to $\bar{\Delta}$. The total space of the family $f^{*} \mathcal{X} \rightarrow \bar{\Delta}$ has double point singularities at the points $p_{i}$ on the central fiber $\left(f^{*} \mathcal{X}\right)_{0}$, which is canonically isomorphic to $X_{0}$. We construct a new family $\overline{\mathcal{X}} \rightarrow \bar{\Delta}$ by blowing-up the singular points on the total space of $f^{*} \mathcal{X}$. Since all the singular points lie on the central fiber, it is an easy check that the fiber $\bar{X}_{\bar{s}}$ is isomorphic to $X_{s}, s=\bar{s}^{2}$, for $\bar{s} \neq 0$. The central fiber $\bar{X}_{0}$ is a normal crossing variety consisting of $v+1$ smooth reduced irreducible components

$$
\bar{X}_{0}=\widehat{X} \cup Q_{1} \cdots \cup Q_{v},
$$

where $\widehat{X} \rightarrow X_{0}$ is the blow-up of $X_{0}$ at the $v$ singular points and $Q_{1}, \ldots, Q_{v}$ are quadric threefolds. The blow-up $\widehat{X} \rightarrow X_{0}$ replaces each ordinary double point $p_{i}$ with an exceptional divisor $E_{i}$ isomorphic to the Hirzebruch surface $\mathbb{F}_{0} . \widehat{X}$ intersects each quadric threefold $Q_{i}$ along $E_{i}$, which is a hyperplane section of $Q_{i}$. Note that the exceptional divisors $E_{i}$ are pairwise disjoint, hence the components $Q_{i}$ have no common points. Therefore, $\bar{X}_{0}$ is indeed a normal crossing divisor with smooth reduced irreducible components.

Note that a small resolution of $X_{0}$ can be obtained by contracting $\widehat{X}$ along a collection of rulings of the exceptional divisor $E_{i} \subset \widehat{X}$. Since each divisor $E_{i}$ admits two distinct rulings, we can obtain in principle different small resolutions $\widetilde{X}$ related by flops. Note however that not all possible contractions result in projective small resolutions. The considerations of this section are valid for any projective contraction $\widehat{X} \rightarrow \widetilde{X}$. 
Next, we will compute the rational homology of the semi-stable model $\bar{X}_{0}$ using Mayer-Vietoris exact sequences. First, we compute the homology of the singular fiber $X_{0}$ in the initial family. From a topological point of view $X_{0}$ can be represented as the cone of a map from a disjoint collection of $v$ three-spheres to a smooth fiber $X_{s}$

$$
f: \sqcup_{i=1}^{v} S_{i}^{3} \longrightarrow X_{s}
$$

so that $f$ maps each $S_{i}^{3}$ homeomorphically onto a vanishing cycle associated to the $i$ th node of $X_{0}$. Therefore $X_{0}$ is homotopy equivalent to the union of $X_{s}$ and $v$ closed four-discs $D_{i}^{4}, i=1, \ldots, 4$ so that $X_{s} \cap D_{i}^{4}=f\left(S_{i}^{3}\right)$. The associated homology Mayer-Vietoris sequence reads

$$
\begin{gathered}
\cdots \longrightarrow \oplus_{i=1}^{v} H_{k}\left(S_{i}^{3}\right) \longrightarrow H_{k}\left(X_{s}\right) \oplus \oplus_{i=1}^{v} H_{k}\left(D^{4}\right) \\
\longrightarrow H_{k}\left(X_{0}\right) \longrightarrow \oplus_{i=1}^{v} H_{k-1}\left(S_{i}^{3}\right) \longrightarrow \cdots
\end{gathered}
$$

In particular we have

$$
0 \longrightarrow H_{4}\left(X_{s}\right) \longrightarrow H_{4}\left(X_{0}\right) \longrightarrow \oplus_{i=1}^{v} H_{3}\left(S_{i}^{3}\right) \longrightarrow H_{3}\left(X_{s}\right) \longrightarrow H_{3}\left(X_{0}\right) \longrightarrow 0
$$

The image of the map $\oplus_{i=1}^{v} H_{3}\left(S_{i}^{3}\right) \rightarrow H_{3}\left(X_{s}\right)$ is the $v-r$ dimensional lattice $V$ of vanishing cycles on $X_{s}$, and the kernel is the $r$-dimensional lattice $R$ of relations among vanishing cycles. Therefore, we find two short exact sequences

$$
\begin{aligned}
& 0 \longrightarrow H_{4}\left(X_{s}\right) \longrightarrow H_{4}\left(X_{0}\right) \longrightarrow R \longrightarrow 0 \\
& 0 \longrightarrow V \longrightarrow H_{3}\left(X_{s}\right) \longrightarrow H_{3}\left(X_{0}\right) \longrightarrow 0
\end{aligned}
$$

which yield the relations $b_{3}\left(X_{0}\right)=b_{3}\left(X_{s}\right)-(v-r), b_{4}\left(X_{0}\right)=b_{4}\left(X_{s}\right)+r$. The remaining Betti numbers can be easily determined by writing down the other terms in (3.2). We record the complete results below, using the notation $b_{k} \equiv b_{k}\left(X_{s}\right)$

\begin{tabular}{lcccccll}
\hline $\mathrm{i}$ & 6 & 5 & 4 & 3 & 2 & 1 & 0 \\
$b_{i}\left(X_{s}\right)$ & 1 & 0 & $b_{2}$ & $b_{3}$ & $b_{2}$ & 0 & 1 \\
$b_{i}\left(X_{0}\right)$ & 1 & 0 & $b_{2}+\mathrm{r}$ & $b_{3}-(v-r)$ & $b_{2}$ & 0 & 1 \\
\hline
\end{tabular}

In order to determine the homology of the blow-up $\widehat{X}$, consider the long exact homology sequence for the pair $(\widehat{X}, E)$

$$
\cdots \longrightarrow H_{k}(E) \longrightarrow H_{k}(\widehat{X}) \longrightarrow H_{k}(\widehat{X}, E) \longrightarrow H_{k-1}(E) \rightarrow \cdots,
$$

where $E=\cup_{i=1}^{v} E_{i}$ denotes the exceptional divisor. Using the fact that $E_{i} \simeq \mathbb{P}^{1} \times \mathbb{P}^{1}$ for each $i=1, \ldots, v$ and that $X_{0}$ is $\widehat{X}$ with each $E_{i}$ collapsed 
to a point, we see that (3.4) reduces to the following straightforward isomorphisms

$$
H_{k}(\widehat{X}) \simeq H_{k}\left(X_{0}\right), \quad k=0,1,5,6
$$

and two exact sequences of the form

$$
\begin{aligned}
& 0 \longrightarrow H_{4}(E) \longrightarrow H_{4}(\widehat{X}) \longrightarrow H_{4}(\widehat{X}, E) \longrightarrow 0 \\
& 0 \longrightarrow H_{3}(\widehat{X}) \longrightarrow H_{3}(\widehat{X}, E) \stackrel{\delta}{\longrightarrow} H_{2}(E) \stackrel{\iota_{*}}{\longrightarrow} H_{2}(\widehat{X}) \longrightarrow H_{2}(\widehat{X}, E) \longrightarrow 0
\end{aligned}
$$

The first sequence yields

$$
b_{4}(\widehat{X})=b_{4}\left(X_{0}\right)+v=b_{2}\left(X_{s}\right)+v+r .
$$

By Poincaré duality we also have

$$
b_{2}(\widehat{X})=b_{4}(\widehat{X})=b_{2}\left(X_{s}\right)+v+r .
$$

Also taking into account (3.3) and the second sequence in (3.5) we find

$$
b_{3}(\widehat{X})=b_{3}\left(X_{s}\right)-2(v-r) \text {. }
$$

Moreover, it follows that

$$
\operatorname{dim}\left(\operatorname{ker}\left(\iota_{*}\right)\right)=v-r, \quad \operatorname{dim}\left(\operatorname{im}\left(\iota_{*}\right)\right)=v+r
$$

where $\iota_{*}: H_{2}(E) \rightarrow H_{2}(\widehat{X})$ is the map on homology determined by the inclu$\operatorname{sion} \iota: E \rightarrow \widehat{X}$. Note that $\operatorname{ker}\left(\iota_{*}\right)=\operatorname{im}(\delta)$ is spanned by relative homology three-cycles for the pair $(\widehat{X}, E)$ modulo cycles in $\widehat{X}$. These cycles generate the lattice of relations among curve classes on $E$ regarded as homology cycles on $\widehat{X}$.

There is a similar exact sequence for the pair $(\tilde{X}, C)$, where $\tilde{X}$ is a small projective resolution of $X_{0}$ and $C$ is the collection of exceptional curves

$$
\cdots \longrightarrow H_{k}(C) \longrightarrow H_{k}(\tilde{X}) \longrightarrow H_{k}(\tilde{X}, C) \longrightarrow H_{k-1}(C) \longrightarrow \cdots
$$

Again, by construction we have $C_{i} \cong \mathbb{P}^{1}$ and $X_{0}$ is $\tilde{X}$ with each $C_{i}$ collapsed to a point. The long exact sequence (3.6) reduces to isomorphisms

$$
H_{k}(\tilde{X})=H_{k}\left(X_{0}\right), \quad k=0,1,4,5,6,
$$

and a shorter exact sequence

$$
0 \longrightarrow H_{3}(\tilde{X}) \longrightarrow H_{3}(\widetilde{X}, C) \stackrel{\widetilde{\delta}}{\longrightarrow} H_{2}(C) \stackrel{\widetilde{\iota}_{*}}{\longrightarrow} H_{2}(\tilde{X}) \longrightarrow H_{2}(\widetilde{X}, C) \longrightarrow 0 .
$$

Then, using (3.7), (3.8) and the fact that $b_{2}(\tilde{X})=b_{4}(\tilde{X})$ one gets

$$
b_{3}(\tilde{X})=b_{3}\left(X_{s}\right)-2(v-r) \text {. }
$$

and

$$
\operatorname{dim}\left(\operatorname{ker}\left(\widetilde{\iota}_{*}\right)\right)=v-r, \quad \operatorname{dim}\left(\operatorname{im}\left(\widetilde{\iota}_{*}\right)\right)=r
$$


where $\widetilde{\iota}_{*}: H_{2}(C) \rightarrow H_{2}(\widetilde{X})$ is the map on homology determined by the inclusion $\widetilde{\iota}: C \rightarrow \widetilde{X}$. Note that $\operatorname{ker}\left(\widetilde{\iota}_{*}\right) \simeq \operatorname{im}(\widetilde{\delta})$ is spanned by relative homology three-cycles on $(\tilde{X}, C)$ modulo cycles in $\tilde{X}$. Just as in the blow-up case these cycles generate the lattice of relations among exceptional curve classes on $\widetilde{X}$.

For future reference, we analyze the direct relation between the rational homology of $\widehat{X}$ and the homology of $\widetilde{X}$. Choose a basis of homology twocycles $\left(a_{i}, b_{i}\right)$ in $H_{2}\left(E_{i}\right), i=1, \ldots, v$ corresponding to the two rulings so that the contraction map $q: \widehat{X} \rightarrow \widetilde{X}$ contracts all rational curves in the classes $b_{i}$, $i=1, \ldots, v$. A simple intersection computation yields the following relations in the homology ring of $\widehat{X}$

$$
a_{i} \cdot E_{j}=b_{i} \cdot E_{j}=-\delta_{i j}, \quad i, j=1, \ldots, v .
$$

Now let us consider the following commutative diagram of spaces

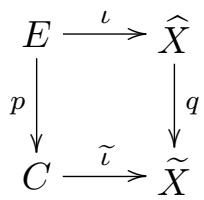

where $p: E \rightarrow C$ is a projection map contracting the rulings $b_{i}, i=1, \ldots, v$. Then we have a natural relation between maps on homology

$$
q_{*} \iota_{*}=\widetilde{\iota}_{*} p_{*} .
$$

This shows that that $p_{*}: H_{2}(E) \rightarrow H_{2}(C)$ induces a map

$$
\bar{p}_{*}: \operatorname{ker}\left(\iota_{*}\right) \longrightarrow \operatorname{ker}\left(\widetilde{\iota}_{*}\right) \text {. }
$$

Moreover, one can show that $\bar{p}_{*}$ is injective, since any element in the kernel of $p_{*}$ is necessarily a linear combination of $b_{i} \in H_{2}(E)$. However, any nontrivial linear combination of $b_{i}$ cannot lie in $\operatorname{ker}\left(\iota_{*}\right)$ because the intersection pairing on $\left(b_{i}, E_{i}\right), i=1, \ldots, v$ is nondegenerate according to (3.9). Therefore $\operatorname{ker}\left(\bar{p}_{*}\right)=0$. Since $\operatorname{ker}\left(\iota_{*}\right), \operatorname{ker}\left(\widetilde{\iota}_{*}\right)$ are $\mathbb{Q}$-vector spaces of equal dimension $v-r$, it follows that $\bar{p}_{*}$ is an isomorphism. Furthermore, $\operatorname{ker}\left(\iota_{*}\right)$ is contained in the linear span of the homology classes $a_{i}-b_{i}, i=1, \ldots, v$.

Note also that the map $q_{*}: H_{3}(\widehat{X}, E) \rightarrow H_{3}(\widetilde{X}, C)$ is an isomorphism. This follows from the commutative diagram of pairs

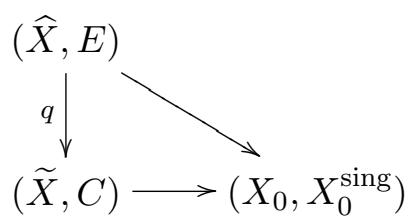


which induces isomorphisms $H_{3}(\widehat{X}, E) \simeq H_{3}\left(X_{0}, X_{0}^{\text {sing }}\right)$ and $H_{3}(\widetilde{X}, C) \simeq$ $H_{3}\left(X_{0}, X_{0}^{\text {sing }}\right)$. Therefore, we obtain the following commutative diagram of homology groups

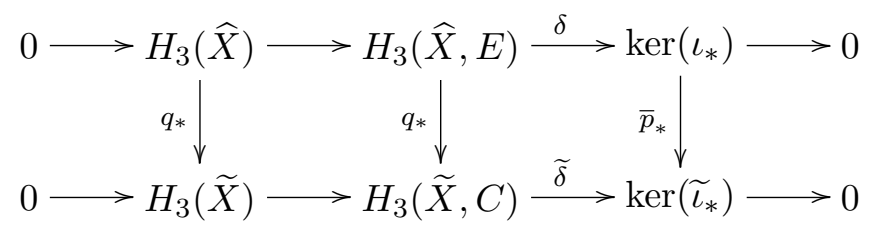

where the rows are exact and the middle and rightmost vertical arrows are isomorphisms. We conclude that the leftmost vertical arrow must be an isomorphism as well, and there is also an isomorphism

$$
H_{3}(\widehat{X}, E) / H_{3}(\widehat{X}) \simeq H_{3}(\tilde{X}, C) / H_{3}(\widetilde{X}) .
$$

To conclude the homology computations, let us determine the homology of the central fiber $\bar{X}_{0}$ of the semistable degeneration. We will employ again a Mayer-Vietoris exact sequence with respect to the closed cover

$$
\bar{X}_{0}=\widehat{X} \cup Q_{1} \cup \cdots \cup Q_{v}
$$

where $Q_{i}$ are smooth quadric threefolds intersecting $\widehat{X}$ transversely along the exceptional divisors $E_{i}, i=1, \ldots, v$. Let $Q$ denote the disjoint union $Q=Q_{1} \cup \ldots \cup Q_{v}$. Then, we have

$$
\begin{aligned}
0 \longrightarrow H_{6}(\widehat{X}) \oplus H_{6}(Q) \longrightarrow H_{6}\left(\bar{X}_{0}\right) \longrightarrow H_{5}(E) \longrightarrow H_{5}(\widehat{X}) \oplus H_{5}(Q) \longrightarrow H_{5}(\bar{X}) \\
\longrightarrow H_{4}(E) \longrightarrow H_{4}(\widehat{X}) \oplus H_{4}(Q) \longrightarrow H_{4}\left(\bar{X}_{0}\right) \longrightarrow H_{3}(E) \longrightarrow H_{3}(\widehat{X}) \oplus H_{3}(Q) \\
\longrightarrow H_{3}(\bar{X}) \longrightarrow H_{2}(E) \longrightarrow H_{2}(\widehat{X}) \oplus H_{2}(Q) \longrightarrow H_{2}\left(\bar{X}_{0}\right) \longrightarrow H_{1}(E) \\
\longrightarrow H_{1}(\widehat{X}) \oplus H_{1}(Q) \longrightarrow H_{1}(\bar{X}) \longrightarrow H_{0}(E) \longrightarrow H_{0}(\widehat{X}) \oplus H_{0}(Q) \\
\longrightarrow H_{0}\left(\bar{X}_{0}\right) \longrightarrow 0
\end{aligned}
$$

The homology of $Q$ can be computed easily from the Lefschetz hyperplane theorem and an Euler characteristic computation via Gauss-Bonet formula. We have

$$
H_{k}(Q)=\mathbb{Q}, \quad k=0,2,4,6, \quad H_{k}(Q)=0, \quad k=1,3,5 .
$$

Moreover, since $E_{i}$ is a hyperplane section of $Q_{i}, i=1, \ldots, v$, it follows that the map

$$
H_{4}(E) \longrightarrow H_{4}(\widehat{X}) \oplus H_{4}(Q)
$$


is injective. Thus, the long exact sequence (3.11) yields the following straightforward isomorphisms

$$
\begin{aligned}
& H_{6}\left(\bar{X}_{0}\right) \simeq H_{6}(\widehat{X}) \oplus H_{6}(Q)=\mathbb{Q}^{\oplus(1+v)} \\
& H_{5}\left(\bar{X}_{0}\right) \simeq H_{5}(\widehat{X}) \oplus H_{5}(Q)=0 \\
& H_{0}\left(\bar{X}_{0}\right) \simeq H_{0}(\widehat{X})=\mathbb{Q} \\
& H_{1}\left(\bar{X}_{0}\right) \simeq H_{1}(\widehat{X})=0 .
\end{aligned}
$$

The remaining part of (3.11) splits into two exact sequences

$0 \longrightarrow H_{4}(E) \longrightarrow H_{4}(\widehat{X}) \oplus H_{4}(Q) \longrightarrow H_{4}\left(\bar{X}_{0}\right) \longrightarrow 0$

$0 \longrightarrow H_{3}(\widehat{X}) \longrightarrow H_{3}\left(\bar{X}_{0}\right) \longrightarrow H_{2}(E) \stackrel{\left(\iota_{*}, j_{*}\right)}{\longrightarrow} H_{2}(\widehat{X}) \oplus H_{2}(Q) \longrightarrow H_{2}\left(\bar{X}_{0}\right) \longrightarrow 0$,

where the $j_{*}: H_{2}(E) \rightarrow H_{2}(Q)$ is the map on homology determined by the inclusion $j: E \hookrightarrow Q$. The first sequence gives

$$
b_{4}(\bar{X})=b_{4}(\widehat{X})+v-v=b_{4}(\widehat{X})=b_{2}\left(X_{s}\right)+v+r .
$$

Since $E$ is a hyperplane section of $Q$, the kernel of the map $j_{*}: H_{2}(E) \rightarrow$ $H_{2}(Q)$ is spanned by the homology classes $a_{i}-b_{i}, i=1, \ldots, v$. On the other hand $\operatorname{ker}\left(\iota_{*}\right)$ is a $v-r$ dimensional subspace of the linear span of $a_{i}-b_{i}$, $i=1, \ldots, v$. Therefore we find that

$$
\operatorname{ker}\left(\iota_{*}, j_{*}\right) \simeq \operatorname{ker}\left(\iota_{*}\right)
$$

is also $v-r$ dimensional. This fixes the remaining Betti numbers

$$
\begin{aligned}
& b_{3}(\bar{X})=b_{3}(\widehat{X})+(v-r)=b_{3}\left(X_{s}\right)-(v-r) \\
& b_{2}(\bar{X})=b_{2}(\widehat{X})+v-(2 v-v+r)=b_{2}\left(X_{s}\right)+v .
\end{aligned}
$$

In summary we can extend the table (3.3) to include all the spaces appearing in the geometric transition and the semi-stable degeneration:

\begin{tabular}{lccccccc}
\hline$i$ & 6 & 5 & 4 & 3 & 2 & 1 & 0 \\
\hline$b_{i}\left(X_{s}\right)$ & 1 & 0 & $b_{2}$ & $b_{3}$ & $b_{2}$ & 0 & 1 \\
$b_{i}\left(X_{0}\right)$ & 1 & 0 & $b_{2}+r$ & $b_{3}-(v-r)$ & $b_{2}$ & 0 & 1 \\
$b_{i}(\widehat{X})$ & 1 & 0 & $b_{2}+v+r$ & $b_{3}-2(v-r)$ & $b_{2}+v+r$ & 0 & 1 \\
$b_{i}(\widetilde{X})$ & 1 & 0 & $b_{2}+r$ & $b_{3}-2(v-r)$ & $b_{2}+r$ & 0 & 1 \\
$b_{i}\left(\bar{X}_{0}\right)$ & $1+v$ & 0 & $b_{2}+v+r$ & $b_{3}-(v-r)$ & $b_{2}+v$ & 0 & 1 \\
$b_{i}(\widetilde{X}, C)$ & 1 & 0 & $b_{2}+r$ & $b_{3}-(v-r)$ & $b_{2}$ & $v-1$ & 1 \\
\hline
\end{tabular}

With all this information in place, we are ready to finish the proof of (ii). 
Recall that

$$
\operatorname{ker}\left(\iota_{*}\right) \simeq H_{3}(\widehat{X}, E) / H_{3}(\widehat{X})
$$

according to the top exact sequence in (3.10). Then the second exact sequence in (3.13) yields a short exact sequence of the form

$$
0 \longrightarrow H_{3}(\widehat{X}) \longrightarrow H_{3}\left(\bar{X}_{0}\right) \longrightarrow H_{3}(\widehat{X}, E) / H_{3}(\widehat{X}) \longrightarrow 0 .
$$

Comparing (3.15) with the top row of (3.10) we get an isomorphism $H_{3}\left(\bar{X}_{0}\right)$ $\cong H_{3}(\widehat{X}, E) / H_{3}(\widehat{X})$ of mixed Hodge structures. Combined with the fact that the vertical arrows in (3.10) are isomorphisms we get an isomorphism $H_{3}\left(\bar{X}_{0}\right) \cong H_{3}(\widetilde{X}, C)$ as mixed Hodge structures. Dualizing and applying the universal coefficients theorem we get the desired isomorphism of mixed Hodge structures $H^{3}\left(\bar{X}_{0}\right) \cong H^{3}(\widetilde{X}, C)$. Since by $(3.12)$ we have $H_{1}\left(\bar{X}_{0}\right)=0$ this completes the proof of (ii).

At this point we still have to tie a few loose ends and prove claim (iii). Note that the universal coefficients theorem and (3.14) give that $H^{5}\left(\bar{X}_{0}\right)=$ 0 . Therefore the Clemens-Schmid exact sequence reduces to

$$
0 \longrightarrow H^{3}\left(\bar{X}_{\bar{s}}\right) / \operatorname{im}(N) \stackrel{\bar{\beta}}{\longrightarrow} H_{3}\left(\bar{X}_{0}\right) \longrightarrow 0 .
$$

The middle map $\bar{\beta}$ in this exact sequence is defined by the sequence of maps

$$
H^{3}\left(\bar{X}_{\bar{s}}\right) \stackrel{P D}{\longrightarrow} H_{3}\left(\bar{X}_{\bar{s}}\right) \longrightarrow H_{3}(\overline{\mathcal{X}}) \simeq H_{3}\left(\bar{X}_{0}\right)
$$

where the first map is Poicaré duality on a smooth fiber and the next map is induced by inclusion in the total space $\overline{\mathcal{X}}$ of the semistable family. Since the image of $N=\log (T)=T$ - id is the space $V$ of vanishing cycles, it follows that we have an isomorphism of mixed Hodge structures

$$
0 \longrightarrow H_{3}\left(\bar{X}_{\bar{s}}\right) / V \longrightarrow H_{3}\left(\bar{X}_{0}\right) \longrightarrow 0 .
$$

The weight filtration on $H_{3}\left(\bar{X}_{\bar{s}}\right) / V$ is a one step filtration induced by the monodromy weight filtration of $H_{3}\left(\bar{X}_{\bar{s}}\right)$

$$
\begin{aligned}
& W_{3}=H_{3}\left(\bar{X}_{\bar{s}}\right) / V \\
& W_{2}=(\operatorname{ker} N) / V=V^{\perp} / V \\
& W_{1}=0
\end{aligned}
$$

Therefore we have a three-term exact sequence

$$
0 \longrightarrow V^{\perp} / V \longrightarrow H_{3}\left(\bar{X}_{\bar{s}}\right) / V \stackrel{p}{\longrightarrow} V^{\vee} \longrightarrow 0
$$

where the map $p$ is induced by $N=\log (T)=T-\mathrm{id}$

$$
p(\bar{\Gamma})=\langle\bar{\Gamma}, \bullet\rangle \text {. }
$$


The monodromy weight filtration on $H_{3}\left(\bar{X}_{0}\right)$ is also a one step filtration of the form

$$
\begin{aligned}
& W_{3}=H_{3}\left(\bar{X}_{0}\right) \simeq H_{3}(\tilde{X}, C) \\
& W_{2}=H_{3}(\widehat{X}) \simeq H_{3}(\widetilde{X}) \\
& W_{1}=0
\end{aligned}
$$

Since (3.16) is an isomorphism of mixed Hodge structures, it is in particular compatible with the weight filtrations. This implies the commutative diagram (2.9) of claim (iii).

\section{Acknowledgments}

We are very grateful to Bogdan Florea and Antonella Grassi for collaboration at an early stage of the project and many useful discussions. The work of D.-E. D. has been partially supported by an Alfred P. Sloan fellowship. R.D. is partially supported by NSF grant DMS 0104354 and FRG grant 0139799 for "The Geometry of Superstrings". T.P. is partially supported by NSF grants FRG 0139799 and DMS 0403884.

\section{References}

[1] R. Gopakumar and C. Vafa, On the gauge theory/geometry correspondence, Adv. Theor. Math. Phys. 3 (1999), 1415.

[2] M. Aganagic and C. Vafa, $G_{2}$ manifolds, mirror symmetry and geometric engineering, 2001, hep-th/0110171.

[3] D.E. Diaconescu, B. Florea and A. Grassi, Geometric transitions and open string instantons, Adv. Theor. Math. Phys. 6 (2002), 619.

[4] D.E. Diaconescu, B. Florea and A. Grassi, Geometric transitions, del Pezzo surfaces and open string instantons, Adv. Theor. Math. Phys. 6 (2002), 643.

[5] M. Aganagic, M. Mariño and C. Vafa, All loop topological string amplitudes from Chern-Simons theory, Comm. Math. Phys. 247 (2004), 467.

[6] D. Diaconescu and B. Florea, Large N duality for compact CalabiYau threefolds, 2003, hep-th/0302076, Adv. Theor. Math. Phys., Nucl. Phys. B 372 (2006), 329-390.

[7] R. Dijkgraaf and C. Vafa, Matrix models, topological strings, and supersymmetric gauge theories, Nucl. Phys. B 644 (2002), 3.

[8] R. Dijkgraaf and C. Vafa, On geometry and matrix models, Nucl. Phys. B 644 (2002), 21. 
[9] S. Donaldson and R. Thomas, Gauge theory in higher dimensions, in 'The geometric universe (Oxford, 1996)', 31-47, Oxford Univ. Press, Oxford, 1998.

[10] E. Witten, Branes and the dynamics of QCD, Nucl. Phys. B 507 (1997), 658.

[11] H. Clemens, Cohomology and obstructions II: Curves on K-trivial threefolds, 2002, math.AG/0206219.

[12] M. Aganagic and C. Vafa, Mirror symmetry, D-branes and counting holomorphic discs, 2000, hep-th/0012041.

[13] W. Lerche, P. Mayr and N. Warner, Holomorphic $N=1$ special geometry of open-closed type II strings, 2002, hep-th/0207259.

[14] W. Lerche, P. Mayr and N. Warner, $N=1$ special geometry, mixed hodge variations and toric geometry, 2002, hep-th/0208039.

[15] B. Forbes, Open string mirror maps from Picard-Fuchs equations on relative cohomology, 2003, hep-th/0307167.

[16] B. Forbes, Computations on B-model geometric transitions, 2004, hepth/0408167.

[17] D.E. Diaconescu et al., Geometric transitions and integrable systems, 2005, preprint.

[18] I. Smith, R. Thomas and S.T. Yau, Symplectic conifold transitions, J. Diff. Geom. 62 (2002), 209.

[19] H. Clemens, Double solids, Adv. Math. 47 (1983), 107.

[20] E. Witten, Mirror manifolds and topological field theory, in 'Essays on mirror manifolds'. Internat. Press, Hong Kong, 1992, 120-158.

[21] R. Bryant and P. Griffiths, Some observations on the infinitesimal period relations for regular threefolds with trivial canonical bundle, in 'Arithmetic and geometry, Vol. II', Progr. Math. 36, Birkhäuser Boston, Boston, MA, 1983, 77-102.

[22] D. Morrison, Mirror symmetry and rational curves on quintic threefolds: a guide for mathematicians, J. Am. Math. Soc. 6 (1993), 223.

[23] D. Freed, Special Kähler manifolds, Comm. Math. Phys. 203 (1999), 31.

[24] E. Witten, Chern-Simons gauge theory as a string theory, in 'The Floer memorial volume', Progr. Math. 133, Birkhäuser, Basel, 1995, 637-678.

[25] P. Griffiths and L. Tu, Variation of Hodge structure, in 'Topics in transcendental algebraic geometry (Princeton, NJ, 1981/1982)', Ann. Math. Stud. 106, Princeton University Press, Princeton, NJ, 1984, 3-28.

[26] W. Schmid, Variation of Hodge structure: the singularities of the period mapping, Invent. Math. 22 (1973), 211. 
[27] P. Deligne, Théorie de Hodge. II, Inst. Hautes Études Sci. Publ. Math. 40 (1971) 5.

[28] P. Deligne, Théorie de Hodge. III, Inst. Hautes Études Sci. Publ. Math. 44 (1974) 5.

[29] H. Clemens, Degeneration of Kähler manifolds, Duke Math. J. 44 (1977), 215. 
